\title{
A Systematic Review of Sustainable Housing Examples: Results Synthesised from the PUBMED and ScienceDirect Databases
}

\author{
Ivy Shiue ${ }^{1 *}$ \\ ${ }^{1}$ School of Energy, Geoscience, Infrastructure \& Society, Heriot-Watt University, Riccarton, EH14 \\ 4AS, Edinburgh, Scotland, UK \\ E-Mail: 1.shiue@hw.ac.uk \\ * Author to whom correspondence should be addressed; Tel.: +44-131-451-4655; Fax: +44-131-451- \\ 1361
}

Received: 3 September 2014 / Accepted: 9 October 2014 / Published: 1 November 2014

\begin{abstract}
Accommodation for both humans and animals could have impacted on both occupants and environments at the same time. Consequently, sustainable housing with a systematic life-cycle assessment has been one of the research focuses in the recent decades with an aim to lessen negative impacts on natural environments and to optimise occupant health and wellbeing. Following this context, it was aimed to carry out a systematic review to synthesise existing literature published until September 2014 on sustainable housing examples from two largest research databases covering health and development research. There were 10 research articles found in the PUBMED database and other 8 research articles additionally found in the ScienceDirect database. Sustainable housing examples mainly came from Americas and Europe while a few were from Africa and Australia. No sound studies were found from Asia. The research quality of these studies was from low to medium only. Research into sustainable housing examples for either humans or animals is still limited and research methodology was not robust enough to give clear indications on the promotion of sustainability in different housing environments. Collaborations between epidemiologists and engineers to employ real-life housing examples and to conduct rigorous research and follow-ups are therefore suggested.
\end{abstract}

Keywords: housing; sustainability; systematic review 


\section{Introduction}

Housing conditions affect occupants continuously and health interventions have shown a positive association between housing investment or improvement and occupant health. ${ }^{1}$ A recent global study has revealed that at least $1 / 4$ residents in every included country, being wealthy or not, would view housing improvement as top priority in the social and political agenda, with a particularly higher percentage in less wealthy countries (i.e. over 60\%; such as in Andorra, Turkey, Ethiopia, Rwanda, and Mali). ${ }^{2}$ In the same survey, it was also found that people who reported poorer health conditions considered housing as top priority ( $\mathrm{OR} 4.56,95 \% \mathrm{CI} 1.10-18.81, \mathrm{P}=0.036)$. Housing problems are prevalent in every society. There were several studies investigating the effects of different housing types on human health and cardiovascular biomarkers, ${ }^{3,4}$ and in particular in children. Moreover, environmental chemicals were found to mediate the effect of old housing on occupant health, ${ }^{5}$ although the sources were not available to be completely investigated. Accommodation for both humans and animals could have impacted on both occupants and environments at the same time. Consequently, sustainable housing with a systematic life-cycle assessment has been one of the research focuses in the recent decades with an aim to lessen negative impacts on natural environments and to optimise occupant health and wellbeing. ${ }^{6}$ Following this context, it was aimed to carry out a systematic review to synthesise existing literature on sustainable housing examples from two largest research databases covering health and development research.

\section{Results and Discussion}

Of all the 110 identified published articles from the PUBMED database using keywords including "sustainability" and "housing", only 10 research articles (published in 2001-2013) met the inclusion criterion (research articles describing housing examples in relation to sustainability) and included for presentation. Table 1 presents publication year, journal, author(s), research question(s), study design and results from each of the included research articles. Critique on study limitations were discussed as well. Overall, the study samples used were small and those sustainable housing examples mainly came from Americas and Europe. Of all the 214 identified published articles from the ScienceDirect database using the same search strategy (some of them were the same found in the PUBMED database), only other 8 research articles (published in 2006-2014) met the inclusion criterion mentioned above and included for presentation. Table 2 also presents publication year, journal, author(s), research question(s), study design and results from each of the included research articles while critique on study limitations were discussed as well. Overall, the study samples used were also small and those sustainable housing examples came from Americas, Europe, Africa and Australia. There were no same housing examples in different published research articles observed.

Table 1. List of the included articles from the PUBMED database.

\begin{tabular}{|c|l|c|c|c|c|}
\hline $\begin{array}{c}\text { Publishing year, } \\
\text { journal and } \\
\text { author(s) }\end{array}$ & Location & $\begin{array}{c}\text { Research } \\
\text { question(s) }\end{array}$ & Methodology & Results & Limitations \\
\hline
\end{tabular}




\begin{tabular}{|c|c|c|c|c|c|}
\hline $\begin{array}{l}2013 \\
\text { Int J Self Help } \\
\text { Self Care. } \\
\text { Harvey, et al. }{ }^{9}\end{array}$ & USA & $\begin{array}{l}\text { What is the } \\
\text { sustainability rate } \\
\text { of self-run } \\
\text { substance abuse } \\
\text { recovery homes? }\end{array}$ & $\begin{array}{l}\text { A longitudinal (6- } \\
\text { year) study } \\
\text { (sample: } 214 \\
\text { houses and } 897 \\
\text { residents) }\end{array}$ & $\begin{array}{l}\text { Sustainability } \\
\text { depends on locale, } \\
\text { primarily access to } \\
\text { affordable housing, } \\
\text { adequate job } \\
\text { opportunities for } \\
\text { residents and } \\
\text { adherence to housing } \\
\text { sustainability rules. }\end{array}$ & $\begin{array}{l}\text { 1) No } \\
\text { investigation on } \\
\text { housing indoor } \\
\text { environment }\end{array}$ \\
\hline $\begin{array}{l}2013 \\
\text { Environ Sci } \\
\text { Technol. } \\
\text { Urban, et al. }{ }^{10}\end{array}$ & USA & $\begin{array}{l}\text { How do } \\
\text { ecosystems fit } \\
\text { into the } \\
\text { sustainability of } \\
\text { residential } \\
\text { systems? }\end{array}$ & $\begin{array}{l}\text { A design- } \\
\text { simulation study }\end{array}$ & $\begin{array}{l}\text { The ecological and } \\
\text { behavioral design } \\
\text { variables were found } \\
\text { to have a significant } \\
\text { effect on carbon } \\
\text { emissions, water } \\
\text { withdrawal and cost } \\
\text { savings. }\end{array}$ & $\begin{array}{l}\text { No presentation } \\
\text { of real-life } \\
\text { housing } \\
\text { examples }\end{array}$ \\
\hline $\begin{array}{l}2012 \\
\text { Rev Panam } \\
\text { Salud Publica. } \\
\text { Eder, et al. }{ }^{11}\end{array}$ & $\begin{array}{l}\text { Potosí } \\
\text { and } \\
\text { Cochaba } \\
\text { mba, } \\
\text { Bolivia }\end{array}$ & $\begin{array}{l}\text { What is the } \\
\text { impact of } \\
\text { Development } \\
\text { Assistance } \\
\text { Program on } \\
\text { maternal and child } \\
\text { health? }\end{array}$ & $\begin{array}{l}\text { A longitudinal (6- } \\
\text { year) intervention } \\
\text { study } \\
\text { (sample: } 12 \\
\text { communities in the } \\
\text { intervention group } \\
\text { and } 2 \text { communities } \\
\text { in the control } \\
\text { group) }\end{array}$ & $\begin{array}{l}\text { The functional } \\
\text { condition of water } \\
\text { system sustained } 42 \% \\
\text { higher in the } \\
\text { intervention group } \\
\text { than in the control } \\
\text { group. }\end{array}$ & $\begin{array}{l}\text { 1) No analysis } \\
\text { on household } \\
\text { size and } \\
\text { composition in } \\
\text { the communities } \\
\text { 2) No } \\
\text { investigation on } \\
\text { the interactions } \\
\text { among water } \\
\text { systems, indoor } \\
\text { air, housing } \\
\text { function and } \\
\text { environmental } \\
\text { impacts }\end{array}$ \\
\hline $\begin{array}{l}2012 \\
\text { J Environ } \\
\text { Manage. } \\
\text { Lee, et al. }{ }^{12}\end{array}$ & $\begin{array}{l}\text { Miami, } \\
\text { USA }\end{array}$ & $\begin{array}{l}\text { How do users' } \\
\text { behaviors affect } \\
\text { residential water- } \\
\text { using appliances? }\end{array}$ & $\begin{array}{l}\text { A life-cycle } \\
\text { assessment study } \\
\text { (sample: } 3 \\
\text { different water- } \\
\text { using appliances) }\end{array}$ & $\begin{array}{l}\text { Earlier replacement } \\
\text { with efficiency } \\
\text { models is encouraged } \\
\text { to minimize the } \\
\text { environmental } \\
\text { impacts of the } \\
\text { product. }\end{array}$ & $\begin{array}{l}\text { 1) Non- } \\
\text { generalisability } \\
\text { of findings } \\
\text { 2) No indications } \\
\text { of any follow-up } \\
\text { monitoring on } \\
\text { water-using } \\
\text { appliances, } \\
\text { housing function } \\
\text { occupant health } \\
\text { and } \\
\text { environmental } \\
\text { impacts }\end{array}$ \\
\hline $\begin{array}{l}2011 \\
\text { Poult Sci. } \\
\text { Lay, et al. }{ }^{13}\end{array}$ & $\begin{array}{l}\text { West } \\
\text { Lafayette, } \\
\text { USA }\end{array}$ & $\begin{array}{l}\text { How do egg } \\
\text { production } \\
\text { systems affect hen } \\
\text { welfare } \\
\text { experience? }\end{array}$ & $\begin{array}{l}\text { A case comparison } \\
\text { study } \\
\text { (sample: } 4 \text { housing } \\
\text { systems) }\end{array}$ & $\begin{array}{l}\text { No single housing } \\
\text { system is ideal from a } \\
\text { hen welfare } \\
\text { perspective. }\end{array}$ & $\begin{array}{l}\text { 1) Non- } \\
\text { generalisability } \\
\text { of findings } \\
\text { 2) No concrete } \\
\text { suggestions on } \\
\text { changing } \\
\text { existing egg } \\
\text { production } \\
\text { systems }\end{array}$ \\
\hline $\begin{array}{l}2010 \\
\text { Sci Total }\end{array}$ & $\begin{array}{l}\text { England, } \\
\text { UK }\end{array}$ & $\begin{array}{l}\text { What are the } \\
\text { energy }\end{array}$ & $\begin{array}{l}\text { A mathematical } \\
\text { study }\end{array}$ & $\begin{array}{l}\text { Some } 96 \% \text { and } 87 \% \\
\text { of energy use and }\end{array}$ & $\begin{array}{l}\text { No presentation } \\
\text { on real-life }\end{array}$ \\
\hline
\end{tabular}




\begin{tabular}{|c|c|c|c|c|c|}
\hline $\begin{array}{l}\text { Environ. } \\
\text { Fidar, et al. }{ }^{14}\end{array}$ & & $\begin{array}{l}\text { consumption and } \\
\text { the environmental } \\
\text { impacts resulting } \\
\text { from water } \\
\text { efficient end uses? }\end{array}$ & & $\begin{array}{l}\text { carbon emissions, } \\
\text { respectively } \\
\text { associated with urban } \\
\text { water provision are } \\
\text { attributable to in- } \\
\text { house consumption } \\
\text { (principally related to } \\
\text { hot water), and that } \\
\text { achieving a defined } \\
\text { water efficiency } \\
\text { target does not } \\
\text { automatically save } \\
\text { energy or reduce } \\
\text { carbon emissions. }\end{array}$ & $\begin{array}{l}\text { housing } \\
\text { examples }\end{array}$ \\
\hline $\begin{array}{l}2010 \\
\text { Sci Total } \\
\text { Environ. } \\
\text { Ortiz-Rodríguez, } \\
\text { et al. }{ }^{15}\end{array}$ & $\begin{array}{l}\text { Pamplona } \\
\text { Colombia } \\
\text { and } \\
\text { Barcelon } \\
\text { a, Spain }\end{array}$ & $\begin{array}{l}\text { What is the } \\
\text { difference } \\
\text { between a } \\
\text { building in } \\
\text { Pamplona, } \\
\text { Colombia and a } \\
\text { building in } \\
\text { Barcelona, Spain } \\
\text { in terms of energy } \\
\text { consumption and } \\
\text { environmental } \\
\text { impacts? }\end{array}$ & $\begin{array}{l}\text { A comparison case } \\
\text { study } \\
\text { (sample: } 2 \\
\text { buildings) }\end{array}$ & $\begin{array}{l}\text { Differences were due } \\
\text { to bio-climatic } \\
\text { differences and the } \\
\text { consumption habits. }\end{array}$ & $\begin{array}{l}\text { 1) No criteria- } \\
\text { matched when } \\
\text { recruiting the } \\
\text { study samples } \\
\text { from } 2 \text { locations } \\
\text { 2) Small study } \\
\text { samples from } 2 \\
\text { locations }\end{array}$ \\
\hline $\begin{array}{l}2008 \\
\text { Environ Manage. } \\
\text { Sorrentino, et } \\
\text { al. }{ }^{16}\end{array}$ & $\begin{array}{l}\text { Pennypac } \\
\text { k Creek } \\
\text { Watershe } \\
\text { d, USA }\end{array}$ & $\begin{array}{l}\text { What is a suitable } \\
\text { housing } \\
\text { placement? }\end{array}$ & $\begin{array}{l}\text { A GIS-based study } \\
\text { (sample: } 2 \\
\text { development } \\
\text { scenarios) }\end{array}$ & $\begin{array}{l}\text { The suitable } \\
\text { development scenario } \\
\text { was } 29 \% \text { better in } \\
\text { terms of energy use } \\
\text { and air and } \\
\text { greenhouse gas } \\
\text { emissions, } 2.4 \% \\
\text { worse on water } \\
\text { quality, and } 2.6 \% \\
\text { better with respect to } \\
\text { biological integrity. }\end{array}$ & $\begin{array}{l}\text { 1) Non- } \\
\text { generalisability } \\
\text { of findings } \\
\text { 2) No indications } \\
\text { of any follow-up } \\
\text { monitoring on } \\
\text { housing function, } \\
\text { occupant health } \\
\text { and } \\
\text { environmental } \\
\text { impacts }\end{array}$ \\
\hline $\begin{array}{l}2006 \\
\text { Br Poult Sci. } \\
\text { Mollenhorst, et } \\
\text { al. }{ }^{17}\end{array}$ & $\begin{array}{l}\text { Wagenin } \\
\text { gen, The } \\
\text { Netherlan } \\
\text { ds }\end{array}$ & $\begin{array}{l}\text { How different } 4 \\
\text { production } \\
\text { systems could } \\
\text { affect sustainable } \\
\text { egg production? }\end{array}$ & $\begin{array}{l}\text { A case study } \\
\text { (sample: unknown } \\
\text { number of hens) }\end{array}$ & $\begin{array}{l}\text { The aviary system } \\
\text { with outdoor run is a } \\
\text { good alternative for } \\
\text { the battery-cage } \\
\text { system, with better } \\
\text { scores for the aviary } \\
\text { system on animal } \\
\text { welfare and } \\
\text { economics, but with } \\
\text { worse scores on } \\
\text { environmental } \\
\text { impact. }\end{array}$ & $\begin{array}{l}\text { 1) Non- } \\
\text { generalisability } \\
\text { of findings } \\
\text { 2) Conflicts of } \\
\text { interests for } \\
\text { animal welfare } \\
\text { and } \\
\text { environmental } \\
\text { impacts }\end{array}$ \\
\hline $\begin{array}{l}2001 \\
\text { Cad Saude } \\
\text { Publica. } \\
\text { Rojas, CA. }{ }^{18}\end{array}$ & $\begin{array}{l}\text { Tumaco, } \\
\text { Colombia }\end{array}$ & $\begin{array}{l}\text { How did the } \\
\text { intervention of an } \\
\text { ecosystem } \\
\text { approach to } \\
\text { human health } \\
\text { have prevented }\end{array}$ & $\begin{array}{l}\text { An intervention } \\
\text { study } \\
\text { (sample: } 10 \\
\text { villages in the } \\
\text { intervention group } \\
\text { and } 23 \text { villages in }\end{array}$ & $\begin{array}{l}\text { Improved housing } \\
\text { and relocation would } \\
\text { decrease the risk of } \\
\text { CL, especially for } \\
\text { children }<5 \text { years. }\end{array}$ & $\begin{array}{l}\text { 1) Small study } \\
\text { sample } \\
\text { 2) No indications } \\
\text { of any follow-up } \\
\text { on housing } \\
\text { function and }\end{array}$ \\
\hline
\end{tabular}




\begin{tabular}{|l|l|l|l|l|}
\hline & $\begin{array}{l}\text { cutaneous } \\
\text { leishmaniasis? }\end{array}$ & the control group) & & $\begin{array}{l}\text { occupant health } \\
\text { monitoring } \\
\text { 3) No indications } \\
\text { of impacts of } \\
\text { new housing } \\
\text { system on } \\
\text { environments }\end{array}$ \\
\hline
\end{tabular}

Table 2. List of the included articles from the ScienceDirect database.

\begin{tabular}{|c|c|c|c|c|c|}
\hline $\begin{array}{l}\text { Publishing year, } \\
\text { journal and } \\
\text { author(s) }\end{array}$ & Location & $\begin{array}{c}\text { Research } \\
\text { question(s) }\end{array}$ & Methodology & Results & Limitations \\
\hline $\begin{array}{l}2014 \\
\text { Energy Build. } \\
\text { Moore T. }{ }^{19}\end{array}$ & $\begin{array}{l}\text { Melbourne, } \\
\text { Australia }\end{array}$ & $\begin{array}{l}\text { What are the } \\
\text { lifecycle cost- } \\
\text { benefits of a new } \\
\text { modelled } \\
\text { housing, } \\
\text { compared to } \\
\text { other housing? }\end{array}$ & $\begin{array}{l}\text { A case } \\
\text { comparison } \\
\text { study } \\
\text { (sample: } 1: 7 \\
\text { housing plans) }\end{array}$ & $\begin{array}{l}\text { There were better } \\
\text { economic and } \\
\text { environment } \\
\text { outcomes presented. }\end{array}$ & $\begin{array}{l}\text { No occupant } \\
\text { health and } \\
\text { wellbeing } \\
\text { investigated }\end{array}$ \\
\hline $\begin{array}{l}2013 \\
\text { Procedia Environ } \\
\text { Sci. } \\
\text { Said, et al. }{ }^{20}\end{array}$ & $\begin{array}{l}\text { Sendangmul } \\
\text { yo, } \\
\text { Indonesia }\end{array}$ & $\begin{array}{l}\text { What is the } \\
\text { environmental } \\
\text { quality of mass } \\
\text { housing? }\end{array}$ & $\begin{array}{l}\text { A survey study } \\
\text { (sample: } 98 \text { out } \\
\text { of } 1828 \\
\text { households) }\end{array}$ & $\begin{array}{l}\text { Good } \\
\text { neighbourhood } \\
\text { interactions, good } \\
\text { sense of belonging, } \\
\text { good maintenance } \\
\text { of residential } \\
\text { infrastructure }\end{array}$ & $\begin{array}{l}\text { 1) Sampling bias } \\
\text { 2) No indications } \\
\text { of any } \\
\text { comparison with } \\
\text { previous } \\
\text { conditions } \\
\text { 3) No indications } \\
\text { of any } \\
\text { intervention and } \\
\text { follow-up on } \\
\text { housing function } \\
\text { and occupant } \\
\text { health monitoring }\end{array}$ \\
\hline $\begin{array}{l}2012 \\
\text { Procedia - Social } \\
\text { Behavioral Sci. } \\
\text { Abdel-Hadi, et } \\
\text { al. }{ }^{21}\end{array}$ & Cairo, Egypt & $\begin{array}{l}\text { What is the } \\
\text { interior } \\
\text { sustainability? }\end{array}$ & $\begin{array}{l}\text { A case study } \\
\text { (sample: } 1 \text { local } \\
\text { community) }\end{array}$ & $\begin{array}{l}\text { The site plan } \\
\text { adhered to the local } \\
\text { environmental } \\
\text { standards. The } \\
\text { awareness of } \\
\text { meeting } \\
\text { environmental } \\
\text { standards towards } \\
\text { sustainability from } \\
\text { residents were } \\
\text { satisfactory. }\end{array}$ & $\begin{array}{l}\text { 1) Non- } \\
\text { generalizability of } \\
\text { findings } \\
\text { 2) No indications } \\
\text { of any } \\
\text { intervention and } \\
\text { follow-up on } \\
\text { housing function } \\
\text { and occupant } \\
\text { health monitoring }\end{array}$ \\
\hline $\begin{array}{l}2012 \\
\text { Habitat Inter. } \\
\text { Sullivan, et al. }{ }^{22}\end{array}$ & USA & $\begin{array}{l}\text { How do we } \\
\text { integrate } \\
\text { sustainable } \\
\text { housing } \\
\text { applications into } \\
\text { self-help and do- } \\
\text { it-yourself } \\
\text { housing }\end{array}$ & $\begin{array}{l}\text { A design- } \\
\text { simulation study }\end{array}$ & $\begin{array}{l}\text { Sustainable housing } \\
\text { applications need to } \\
\text { go with community } \\
\text { and social } \\
\text { organisational } \\
\text { development as well } \\
\text { as fiscal and } \\
\text { juridical policy }\end{array}$ & $\begin{array}{l}\text { No presentation } \\
\text { on real-life } \\
\text { housing examples }\end{array}$ \\
\hline
\end{tabular}




\begin{tabular}{|c|c|c|c|c|c|}
\hline & & $\begin{array}{l}\text { construction and } \\
\text { home } \\
\text { improvements? }\end{array}$ & & dimensions. & \\
\hline $\begin{array}{l}2011 \\
\text { Procedia } \\
\text { Engineering. } \\
\text { Roodgar, et al. }{ }^{23}\end{array}$ & Kashan, Iran & $\begin{array}{l}\text { What can be } \\
\text { improved for } \\
\text { sustainable } \\
\text { development in } \\
\text { future green } \\
\text { buildings in } \\
\text { terms of water } \\
\text { and energy } \\
\text { consumption and } \\
\text { environmental } \\
\text { design? }\end{array}$ & $\begin{array}{l}\text { A case study } \\
\text { (sample: } 1 \\
\text { ancient mansion) }\end{array}$ & $\begin{array}{l}\text { The air gaps in the } \\
\text { external walls can } \\
\text { be replaced by foam } \\
\text { insulation. An } \\
\text { additional layer of } \\
\text { polyurethane } \\
\text { insulation can be } \\
\text { added to the roof. } \\
\text { The use of high } \\
\text { thermal inertia } \\
\text { walls, with excellent } \\
\text { thermal insulation, } \\
\text { in buildings would } \\
\text { usually result in a } \\
\text { reduction of energy } \\
\text { requirements. }\end{array}$ & $\begin{array}{l}\text { 1) Non- } \\
\text { generalizability of } \\
\text { findings } \\
\text { 2) No occupant } \\
\text { health and } \\
\text { wellbeing } \\
\text { investigated }\end{array}$ \\
\hline $\begin{array}{l}2009 \\
\text { Cities. } \\
\text { Howley, et al. }{ }^{24}\end{array}$ & $\begin{array}{l}\text { Dublin, } \\
\text { Ireland }\end{array}$ & $\begin{array}{l}\text { What are the } \\
\text { residential } \\
\text { preferences } \\
\text { towards new } \\
\text { residential } \\
\text { development? }\end{array}$ & $\begin{array}{l}\text { A survey study } \\
\text { (sample: } 50 \\
\text { households) }\end{array}$ & $\begin{array}{l}\text { More space, cleaner } \\
\text { environment, less } \\
\text { noise and better } \\
\text { facilities for } \\
\text { children were } \\
\text { preferred for a } \\
\text { sustainable } \\
\text { residential } \\
\text { development. }\end{array}$ & $\begin{array}{l}\text { 1) Sampling bias } \\
\text { 2) No } \\
\text { investigation of } \\
\text { the linkage } \\
\text { between the } \\
\text { change of } \\
\text { residential } \\
\text { development and } \\
\text { residents' } \\
\text { preferences }\end{array}$ \\
\hline $\begin{array}{l}2008 \\
\text { Build environ. } \\
\text { Isik, et al. }\end{array}$ & $\begin{array}{l}\text { Northern } \\
\text { Cyprus }\end{array}$ & $\begin{array}{l}\text { How does Alker } \\
\text { as a wall material } \\
\text { contribute to } \\
\text { sustainable } \\
\text { development? }\end{array}$ & $\begin{array}{l}\text { A descriptive } \\
\text { study } \\
\text { (sample: } 5 \text { types } \\
\text { of exterior walls } \\
\text { from unknown } \\
\text { number of } \\
\text { historical } \\
\text { buildings) }\end{array}$ & $\begin{array}{l}\text { Alker as a wall } \\
\text { material, with its } \\
\text { low embodied } \\
\text { energy, low heat } \\
\text { transfer value, and } \\
\text { significant health } \\
\text { advantages, could } \\
\text { make a major } \\
\text { contribution toward } \\
\text { sustainable housing. }\end{array}$ & $\begin{array}{l}\text { 1) No time-series } \\
\text { analysis carried } \\
\text { out } \\
\text { 2) No occupant } \\
\text { health and } \\
\text { wellbeing } \\
\text { investigated }\end{array}$ \\
\hline $\begin{array}{l}2006 \\
\text { Agri Ecosys } \\
\text { Environ. } \\
\text { Castellini, et al. }{ }^{26}\end{array}$ & Italy & $\begin{array}{l}\text { What farming } \\
\text { system is better } \\
\text { with environment } \\
\text { sustainability in } \\
\text { terms of energy } \\
\text { use? }\end{array}$ & $\begin{array}{l}\text { A comparative } \\
\text { study } \\
\text { (sample: } 2 \\
\text { farming systems) }\end{array}$ & $\begin{array}{l}\text { The organic farming } \\
\text { system with a } \\
\text { higher efficiency in } \\
\text { transforming the } \\
\text { available inputs in } \\
\text { the final product, a } \\
\text { higher level of } \\
\text { renewable inputs, a } \\
\text { higher level of local } \\
\text { inputs and a lower } \\
\text { density of energy } \\
\text { and matter flows. }\end{array}$ & $\begin{array}{l}\text { 1) Non- } \\
\text { generalizability of } \\
\text { findings } \\
\text { 2) No } \\
\text { investigation of } \\
\text { animal welfare }\end{array}$ \\
\hline
\end{tabular}


In Table 3, summary scores of research quality for each included research article were calculated and are shown separately. Overall, the research quality was low to medium only. The main problems were lack of clear description on analysis process and a vigorous research methodology.

Table 3. Summary scores of research quality for each included research article.

\begin{tabular}{|c|c|c|}
\hline & Summary Scores & Quality \\
\hline (2013) Int J Self Help Self Care. Harvey, et al. ${ }^{9}$ & $8 / 22=36.4 \%$ & low \\
\hline (2013) Environ Sci Technol. Urban, et al. ${ }^{10}$ & $7 / 18=38.9 \%$ & low \\
\hline (2012) Rev Panam Salud Publica. Eder, et al. ${ }^{11}$ & $8 / 22=36.4 \%$ & low \\
\hline (2012) J Environ Manage. Lee, et al. ${ }^{12}$ & $7 / 18=38.9 \%$ & low \\
\hline (2011) Poult Sci. Lay, et al. ${ }^{13}$ & $7 / 18=38.9 \%$ & low \\
\hline (2010) Sci Total Environ. Fidar, et al. ${ }^{14}$ & $6 / 12=50 \%$ & medium \\
\hline (2010) Sci Total Environ. Ortiz-Rodríguez, et al. ${ }^{15}$ & $8 / 18=44.4 \%$ & medium \\
\hline (2008) Environ Manage. Sorrentino, et al. ${ }^{16}$ & $7 / 18=38.9 \%$ & low \\
\hline (2006) Br Poult Sci. Mollenhorst, et al. ${ }^{17}$ & $7 / 18=38.9 \%$ & low \\
\hline (2001) Cad Saude Publica. Rojas, CA. ${ }^{18}$ & $12 / 28=42.9 \%$ & medium \\
\hline (2014) Energy Build. Moore T. ${ }^{19}$ & $5 / 18=27.8 \%$ & low \\
\hline (2013) Procedia Environ Sci. Said, et al..$^{20}$ & $10 / 28=35.7 \%$ & low \\
\hline (2012) Procedia - Social Behavioral Sci. Abdel-Hadi, et al. ${ }^{21}$ & $6 / 18=33.3 \%$ & low \\
\hline (2012) Habitat Inter. Sullivan, et al. ${ }^{22}$ & $7 / 18=38.9 \%$ & low \\
\hline (2011) Procedia Engineering. Roodgar, et al. ${ }^{23}$ & $5 / 18=27.8 \%$ & low \\
\hline (2009) Cities. Howley, et al. ${ }^{24}$ & $8 / 22=36.4 \%$ & low \\
\hline (2008) Build environ. Isik, et al. ${ }^{25}$ & $5 / 18=27.8 \%$ & low \\
\hline (2006) Agri Ecosys Environ. Castellini, et al. ${ }^{26}$ & $6 / 18=33.3 \%$ & low \\
\hline
\end{tabular}

\section{Experimental Section}

The present study employed a systematic review approach, which is used to provide research evidence for future research and policy use. According to Khan, et al, ${ }^{7}$ a review earns the adjective systematic if it is based on a clearly formulated question, identifies relevant studies, appraises their quality and summarises the evidence by use of explicit methodology. Following this framework, the proposed 5 steps, namely "Framing questions for a review", "Identifying relevant work", "Assessing the quality of studies", "Summarising the evidence" and "Interpreting the findings", of conducting a systematic review were therefore adopted in the current study. Analysis of quality of the included quantitative and qualitative studies was based on the assessment checklists proposed by Kmet, et al. ${ }^{8}$ For quantitative studies, there are 14 criteria to score each article. For qualitative studies, there are 10 
criteria. For each criterion, if an article fully met, then the score would be 2 . However, if an article only partially met, the score would be 1 . No score would be given if an article did not meet the criterion. Following this calculation, the maximum scores would be 28 and 20 for quantitative and qualitative studies, respectively.

The inclusion criterion of literature was research articles describing housing examples in relation to sustainability. Therefore, keywords used were "sustainability" and "housing". Other articles dealing with parts of housing system from an engineering perspective were excluded. Since this study is only a literature search and synthesis by extracting published research articles from two largest research databases, namely PUBEMD and ScienceDirect, covering health and development research until September 2014, no further ethics approval was required.

\section{Conclusions}

The included research articles from the current systematic review are fewer than expectation, given that the sustainability has been one of the research focuses in recent years. Sustainable housing examples in research articles were published only since 2001. Although there could have been some proposed sustainability indexes from environmental scientists, engineers or architects, ${ }^{27,28}$ those have not been systematically tested in real life. In addition, most of the articles describing housing system and environmental sustainability did not mention the relationships among housing repair, occupant wellbeing and environmental sustainability. In other words, it is not known whether the change of housing system could actually bring more repair that would chronically and cumulatively result in financial harm and environmental damage. In sum, research into sustainable housing examples for either humans or animals is still limited and research methodology was not robust enough to give clear indications on the promotion of sustainability in different housing environments. Collaborations between epidemiologists and environmental professionals to conduct rigorous research and follow-ups on improving sustainable housing and occupant health in real life in the next decades are strongly suggested.

\section{Acknowledgments}

IS is supported by the Global Platform for Research Leaders scheme.

\section{Conflict of Interest}

The author declares no conflict of interest.

\section{References}

1. Thomson H, Thomas S, Sellstrom E, Petticrew M. Housing improvements for health and associated socio-economic outcomes. Cochrane Database of Systematic Reviews. 2003;No.: CD008657. DOI: 10.1002/14651858.CD008657.pub2.

2. Shiue I. Public perceptions of housing improvement and self-rated health: World Values Survey, 2005-2007. Housing, Care and Support. 2014;17:2

3. Jacobs DE, Wilson J, Dixon SL, Smith J, Evens A. The relationship of housing and population health: A 30-year retrospective analysis. Environ Health Perspect. 2009;117:597-604. 
4. Shiue I, Shiue YY. The role of housing characteristics in biomarkers: US NHANES, 20032006. Int J Cardiol. 2013; dx.doi.org/10.1016/j.ijcard.2013.07.200.

5. Shiue I, Bramely G. Environmental chemicals mediated the effect of old housing on adult health problems: US NHANES, 2009-2010. Environ Sci Pollut Res Int. 2014;doi:10.1007/s11356-014-3468-5.

6. Xin H, Gates RS, Green AR, Mitloehner FM, Moore PA Jr, Wathes CM. Environmental impacts and sustainability of egg production systems. Poult Sci. 2011;90:263-277.

7. Khan KS, Kunz R, Kleijnen J, Antes G. Five steps to conducting a systematic review. J R Soc Med. 2003;96:118-121.

8. Kmet LM, Lee RC, Cook LS. Standard quality assessment criteria for evaluating primary research papers from a variety of fields. HTA Initiative. 2004. (http://www.ihe.ca/documents/HTA-FR13.pdf)

9. Harvey R, Mortensen J, Aase D, Ferrari JR, Jason L. Factors Affecting the Sustainability of Self-Run Recovery Homes in the United States. Int J Self Help Self Care. 2013;7:99-109.

10. Urban RA, Bakshi BR. Techno-ecological synergy as a path toward sustainability of a North American residential system. Environ Sci Technol. 2013;47:1985-1993.

11. Eder C, Schooley J, Fullerton J, Murguia J. Assessing impact and sustainability of health, water, and sanitation interventions in Bolivia six years post-project. Rev Panam Salud Publica. 2012;32:43-48.

12. Lee M, Tansel B. Life cycle based analysis of demands and emissions for residential waterusing appliances. J Environ Manage. 2012;101:75-81.

13. Lay DC Jr, Fulton RM, Hester PY, Karcher DM, Kjaer JB, Mench JA, Mullens BA, Newberry RC, Nicol CJ, O'Sullivan NP, Porter RE. Hen welfare in different housing systems. Poult Sci. 2011;90:278-294.

14. Fidar A, Memon FA, Butler D. Environmental implications of water efficient microcomponents in residential buildings. Sci Total Environ. 2010;408:5828-5835.

15. Ortiz-Rodríguez O, Castells F, Sonnemann G. Life cycle assessment of two dwellings: one in Spain, a developed country, and one in Colombia, a country under development. Sci Total Environ. 2010;408:2435-2443.

16. Sorrentino JA, Meenar MM, Flamm BJ. Suitable housing placement: a GIS-based approach. Environ Manage. 2008;42:803-820.

17. Mollenhorst H, Berentsen PB, De Boer IJ. On-farm quantification of sustainability indicators: an application to egg production systems. Br Poult Sci. 2006;47:405-417.

18. Rojas CA. An ecosystem approach to human health and the prevention of cutaneous leishmaniasis in Tumaco, Colombia. Cad Saude Publica. 2001;17 Suppl:193-200.

19. Moore T. Modelling the through-life costs and benefits of detached zero (net) energy housing in Melbourne, Australia. Energy Build. 2014;70:463-471.

20. Said FRD, Yuliastuti N. Mass housing sustainability based on community cohesion (A case study at Sendangmulyo, Indonesia). Procedia Environ Sci. 2013;17:814-821. 
21. Abdel-Hadi A, Aboulgheit I. Assessing housing interior sustainability in a sew Egyptian city. Procedia - Social Behavioral Sci. 2012;68:564-577.

22. Sullivan E, Ward PM. Sustainable housing applications and policies for low-income self-build and housing rehab. Habitat International. 2012;36:312-323.

23. Roodgar M, Mahmoudi MM, Ebrahimi P, Molaei D. Sustainability, architectural topology and green building evaluations of Kashan-Iran as a hot-arid region. Procedia Engineering. 2011;21:811-819.

24. Howley P, Scott M, Redmond D. An examination of residential preferences for less sustainable housing: Exploring future mobility among Dublin central city residents. Cities. 2009;26:1-8.

25. Isik B and Tulbentci T. Sustainable housing in island conditions using Alker-gypsum-stabilized earth: A case study from northern Cyprus. Build environ. 2008;43:1426-1432.

26. Castellini C, Bastianoni S, Granai C, Bosco AD, Brunetti M. Sustainability of poultry production using the emergy approach: Comparison of conventional and organic rearing systems. Agri Ecosys Environ. 2006;116: 343-350.

27. Russell, PAG. Housing design in the pursuit of sustainability. Proceedings of the Air \& Waste Management Association's Annual Meeting \& Exhibition, 1996.

28. Gray NF, Carton-Kenney M. A rural housing sustainability index. Proceedings of the Institution of Civil Engineers: Municipal Engineer. 2004;157:275-283.

(C) 2014 by the authors; licensee MDPI, Basel, Switzerland. This article is an open access article distributed under the terms and conditions of the Creative Commons Attribution license. 